\title{
Mutations et articulations contemporaines des industries culturelles
}

Pierre-Jean Benghozi

Création et diversité au miroir des industries culturelles J OURNEES D'ECONOMIE DE LA CULTURE 12-13 J ANVIER 2005

Pole de Recherche en Economie et gestion de l'Ecole Polytechnique (UMR

CNRS 7176)

1, rue Descartes - 75005 Paris France

Tel. (33) 0155558447 - Fax (33) 015555844 - Pierre-

Jean.Benghozi@shs.polytechnique.fr 


\section{Résumé}

Les industries culturelles sont à la fois à la base de création de richesses marchandes et sources de valeur symbolique et identitaire. A ce titre, elles focalisent de puissants enjeux dont témoignent, au niveau national ou international, les débats récents. Situer ces enjeux économiques apparaît, dès lors, essentiel pour comprendre les controverses actuelles et guider l'action publique.

La première caractéristique, presque triviale, des industries culturelles est qu'elles reposent sur une part déterminante de création artistique. Cette composante "culturelle » des industries de la culture appelle, d'une part, une articulation constante avec l'ensemble du secteur culturel. Elle pèse d'autre part très directement sur la nature économique des produits culturels et sur leurs modes de production. Elle place en particulier les industries de la culture dans une situation de risque inhabituelle dans les industrie plus classiques. La seconde caractéristique, tout aussi naturelle, des industries de la culture est leur nature industrielle et technologique. I/ s'agit, substantiellement, d'industries de la reproduction, définies par la capacité de dupliquer, via la technologie, des créations singulières. Ce poids de la reproduction explique le rôle important, dans son économie, du facteur technologique tout comme celui de la distribution. De ce point de vue, les industries de la culture ont développé des stratégies industrielles très classiques en travaillant leurs création pour échapper à leur singularité et à leur unicité.

Après avoir posé ces caractéristiques, la communication présentée analyse les mutations et articulations contemporaines des industries culturelles en en dégageant les traits les plus notables. L'ancienneté de ces industries inscrit leur économie dans la durée et éclaire les situations observables aujourd'hui ainsi que les stratégies et réponses spontanément mises en œuvres. Les industries sont marquées par une surproduction généralisée, une spirale inflationniste des coûts et une focalisation des consommations dans une logique de star system qui conduit à rendre particulièrement centrales les fonctions de programmation et de prescription assurées par les diffuseurs. Les industries de la culture sont marquées par une variabilité et une superposition permanente de modèles économiques différents qui conduit à des marchés à la fois plus segmentés et plus diversifiés et qui contribue à l'effacement des frontières traditionnelles de ces industries.

L'ensemble de ces dynamiques rendent particulièrement sensibles et difficiles la régulation et l'action publique dans ce domaine 


\section{Introduction}

Les industries culturelles sont à la fois à la base de création de richesses marchandes et la première source de valeur symbolique et identitaire. A ce titre, elles focalisent de puissants enjeux dont témoignent les nombreux débats récents, au niveau national comme international: convention sur la diversité culturelle, projets de numérisation des bibliothèques, légalité et rémunération des échanges de fichiers... Situer les enjeux économiques de ces industries apparaît, dès lors, essentiel pour comprendre comment ces débats se posent aujourd'hui dans l'espace public et pour éclairer la définition de solutions d'action éventuelles du point de vue de l'acteur public.

La nature particulière des industries culturelles tient à une double spécificité qui fait que d'aucuns en ont parlé comme d'«industries de l'imaginaire ${ }^{1}$. Pour rendre compte du champ de plus en plus large de ce secteur, on évoque même désormais fréquemment le terme d'industries créatives, en soulignant principalement le poids de certains traits traditionnels de la culture (Caves 2000; Throsby 2001) et en mettant en avant les caractéristiques économiques spécifiques aux activités de création (Schussman et Healy 2002) : droits de la propriété (Howkins 2001), gestion du talent et compétences (Tulgan 2001; Florida 2002), externalités (Keat 2000; Throsby 2001), et politiques publiques (Chandler, Hagstrom et al. 1998; Braczyk, Fuchs et al. 1999; Landry 2000; Santagata 2002).

Dans le cadre limité de cette communication, nous avons choisi, pour notre part, de caractériser les traits saillants qui nous semblent marquer aujourd'hui les industries culturelles d'abord, d'identifier les mutations et articulations contemporaines de ce secteur ensuite, d'envisager en conclusion les difficultés rencontrées, à ce titre, par l'action publique.

\section{Des industries à base de création}

La première caractéristique, presque triviale, des industries culturelles est leur nature culturelle, reposant sur une part déterminante de création artistique originale.

\section{QUALITE ET VARIETE DES CEUVRES}

Cette spécificité pèse d'abord très directement sur la manière dont les produits culturels sont appréhendés et consommés. En effet, ils n'assurent pas simplement des besoins fonctionnels mais offrent d'abord un contenu d'ordre immatériel. La valeur symbolique des produits culturels est ainsi bien plus élevée que leur valeur d'usage et est souvent sans lien avec leur coût de production. Alors que la plupart des biens industriels se caractérisent par leurs caractéristiques formelles (vitesse, consommation, taille, confort par exemple pour une voiture) et que leur valeur est en lien directe avec leur prix de fabrication, les produits culturels s'inscrivent dans un cadre où la valeur d'un disque ou un film pour les consommateurs n'est pas définie par le temps d'écoute ou le nombre d'interprètes, pas plus

1 Flichy, P. (1991). Les Industries de l'Imaginaire, Presses Universitaires de Grenoble 
que par le coût de la production ${ }^{2}$, mais essentiellement par le prix attaché à la qualité perçue des auteurs, de l'interprétation et de l'œuvre.

Cette situation contribue à homogénéiser les prix des biens culturels indépendamment de leur qualité et de leur coût intrinsèque. L'économie de la production de tels biens s'inscrit en effet dans un cadre, bien documenté dans la littérature économique, où les coûts fixes (investissements de production et infrastructures) sont importants et les prix de référence sont ceux définis par les structures de distribution selon leur « format » de consommation (place de cinéma, $C D$, livre, cassette vidéo...).

Par exemple, le prix d'une place de cinéma et le réseau de salles seront les mêmes pour une superproduction et un film d'indépendant dont le coût peut être cent fois moindre. Dans ce contexte, les producteurs ont du mal à valoriser de façon différenciée des pratiques de productions relevant d'économies différentes mais s'inscrivant dans des canaux de diffusion communs : la stratégie commerciale de promotion fait alors souvent la différence et, comme nous le verrons plus loin, cristallise l'essentiel des enjeux. Ainsi, dans le cinéma, les distributeurs optimisent les sorties de films en occupant le plus grand nombre d'écrans. Cette stratégie renchérit les coûts de distribution et induit un effet inflationniste ${ }^{3}$ où sauf en cas de succès immédiat, le faible nombre de diffusions ne permet pas d'amortir les coûts de production ni même parfois de diffusion mais permet de maximiser l'effet des campagnes de promotion. Le même phénomène est observable, dans des termes analogues, dans le secteur de l'édition littéraire.

\section{DES CREATI ONS PAR NATURE RI SQUEES}

La seconde conséquence importante du poids de la composante créative tient au degré très important de risque que comportent les industries culturelles. Contrairement à la plupart des autres biens marchands, les produits culturels sont, par nature, le résultat de créations originales dont le succès s'avère éminemment aléatoire. Quels que soient les efforts de promotion, le soin apporté à la création, la renommée des contributeurs ou l'attractivité supposée du thème de l'œuvre, le public n'est pas toujours au rendez vous et les exemples abondent de productions ambitieuses ayant connu des échecs retentissants. Certes, la possibilité d'échec existe dans toute activité industrielle, mais l'ampleur de l'insuccès ${ }^{4}$ n'a en général aucun rapport avec ceux que l'on peut rencontrer dans les industries culturelles.

Ce caractère très incertain de la réussite est d'ailleurs à la base des modes de rémunérations originaux mis en place dans ce secteur et reposant très fortement sur des mécanismes (droits d'auteurs, royautés) permettant à la fois de protéger les producteurs (en évitant des rémunérations hors de proportion en cas d'échec) et de rétribuer correctement les contributeurs en cas de succès.

Comme nous le verrons plus loin, ce caractère très risquée des productions a bien sûr des conséquences quant aux stratégies déployées par les producteurs et les diffuseurs, dans le cadre de ce que l'on a pu appeler une « économie -casino » spéculative. II pèse également très directement sur la focalisation des consommateurs sur un nombre d'œuvres qui va en se restreignant.

\footnotetext{
${ }^{2}$ Même si la médiatisation des produit culturels tend de plus en plus à faire de ces coûts un facteur de promotion en soi.

${ }^{3}$ Ce phénomène couplant surqualité et creusement des déficits a été qualifié, dans le spectacle vivant, de "festival permanent". Greffe, X. et X. Dupuis (1981). "Quand l'opéra découvre la gestion." Revue Française de Gestion 30.

${ }^{4}$ Tout comme, d'ailleurs, le niveau parfois extraordinaire du succès
} 


\section{DES PRODUCTI ONS CREATIVES}

Le poids de la création repose aussi dans les conditions de production initiales ainsi que dans les conditions du passage des œuvres de création aux produits culturels. II suppose des modes de gestion et de production assurant une valorisation économique sur grande échelle tout en préservant la singularité des contributions artistiques. C'est dans cette tension que réside, paradoxalement, les limites d'une industrialisation excessive. Une rationalisation trop systématique de la production et de la diffusion tend en effet à gommer toute originalité et toute prise de risque créatif, conduisant ipso facto à une banalisation des produits culturels risquant de leur ôter tout attrait. La gestion de cette tension est d'autant plus difficile que les industriels comme les professionnels tendent à dissocier la composante créative et le rôle des artistes d'un côté, les formes d'organisation et économiques encadrant la création de l'autre côté. Or toutes les études montrent qu'aujourd'hui la création est essentiellement collective et que les productions culturelles obtenues ne se réduisent que rarement à la seul étincelle créative d'un auteur. La création est de plus en plus le résultat d'interactions répétées d'agents plongés dans une chaîne de valeur globale.

Un des éléments clés de cette chaîne de valeur tient, en particulier, au poids de la fonction éditoriale et de programmation. Dans un contexte risqué qui semble a priori défier les lois du marketing, les intermédiaires assurant, auprès des créateurs, le relais du marché sont particulièrement importants qu'il s'agissent de producteurs de cinéma, de directeurs artistiques dans l'industrie phonographique ou d'éditeurs littéraires. Dans des industries où les contenus sont déterminants, ils contribuent en effet, sur la base de leur perception des consommateurs, à sélectionner les projets, choisir les contributeurs artistiques, accompagner l'élaboration de l'œuvre. Ce faisant, ils dépassent leur seul rôle en production dans la mesure où ils assurent, plus largement, une fonction de mise en avant de certains produits auprès des consommateurs.

En effet, les produits culturels sont essentiellement ce que les économistes appellent des biens d'expérience, à savoir des biens dont on ne peut identifier la qualité tant que le bien n'est pas consommé : impossible de connaître la valeur d'un livre tant qu'on ne l'a pas lu, ni celle d'un film tant qu'on ne l'a pas vu, a fortiori quand l'œuvre ou les auteurs sont mal connus. Dans de tels cas d'information imparfaite, le consommateur a beaucoup de mal à sélectionner les produits qu'il souhaite acquérir. II doit, pour ce faire, s'appuyer sur des éléments, extérieurs au produit, lui permettant de guider son choix : bouche à oreille, lecture de critiques spécialisés, labels de qualité ${ }^{5}$. Le programmateur et l'éditeur, au sens générique du terme, constituent également de tels certificateurs potentiels de qualité. Par la constance de leurs choix, ils peuvent garantir au consommateur un type et un niveau de qualité attendue relativement stables: qu'il s'agisse de la collection blanche de Gallimard, de telle radio ou label musical spécialisé dans un genre donné. Cette fonction de marque apparaît d'autant plus importante que, comme nous le verrons plus loin, les industries culturelles sont marquées par un volume de production grandissant, accru encore avec les œuvres désormais disponibles sur l'internet.

\section{DES INDUSTRI ES QUI S'ARTI CULENT AVEC LE MI LI EU CULTUREL}

Les industries culturelles s'inscrivent d'une part de plein pied dans un secteur marchand très internationalisé, mais elles s'appuient, d'autre part, sur un tissu diversifié de relations noués avec un secteur culturel marqué par le poids de structures de petites tailles dont les enjeux

\footnotetext{
${ }^{5}$ pensons aux diapasons d'or ou aux ffff de Télérama dont les sticks peuvent garnir les CD musicaux.
} 
économiques sont d'une toute autre nature (organisation d'ampleur modeste, structures non lucratives, marchés locaux). Cette situation conduit à une organisation multipolaire des marchés, marquée notamment par des transferts de charge, de responsabilités et de compétences entre les industries de la culture d'un côté, le milieu culturel au sens large de l'autre côté. On en retrouve les conséquences à plusieurs niveaux, notamment dans le marché du travail, dans celui de la production et dans la distribution.

Tout d'abord, les industries culturelles s'appuient, dans une très large mesure, sur des contributeurs artistiques (créateurs et techniciens) relevant d'un emploi intermittent essentiellement organisé, régulé et financé à partir d'un modèle issu du spectacle vivant. La situation est le plus manifeste pour les musiciens alternant enregistrements phonographiques et concerts. Elle est du même ordre pour les comédiens et techniciens travaillant souvent pour le cinéma et le théâtre. Les raisons de cette situation tiennent à un souci légitime de pouvoir diversifier les sources de création, d'assurer un renouvellement régulier des productions en s'appuyant sur les structures émergentes à la frange du marché assurant le repérage des auteurs, des créateurs et des courants artistiques. Elles n'entraînent pas moins des conséquences importantes pour l'organisation des filières culturelles.

Du point de vue individuel, l'imbrication des activités conduit d'abord une grande part des contributeurs artistiques à organiser leur rémunération et gérer leur activité dans une spécialisation risquée ou en assurant un polyvalence et un va et vient entre les deux pôles industriels et pratiques vivantes (spectacle mais aussi enseignement). Du point de vue des entreprises, la situation conduit à l'absence de prise en charge d'une véritable gestion du personnel artistique (carrière, formation, évolution, responsabilité) ${ }^{6}$, en allégeant d'autant la structure de l'entreprise et en reportant sur l'ensemble du secteur des coûts d'ajustement du marché du travail qui, dans les autres industries, sont directement pris en charge par les firmes. Comme l'ont souligné les récents événements qui ont secoué ce secteur, il faut sans doute voir là une des raisons majeures de la crise du financement de l'intermittence.

Du point de vue de la filière enfin, cette situation peut se traduire, comme nous le verrons plus loin, par des formes de concurrence plus ou moins explicites entre secteurs industriels et non marchand, associatif et professionnel, bénévole et salarié. Cette concurrence n'est pas seulement une concurrence entre les œuvres produites; ce peut être aussi une concurrence pour le contrôle des artistes à succès potentiels; c'est aussi une concurrence pour l'accès à la notoriété, aux canaux de distribution et de diffusion.

\section{La dimension industrielle}

Si le fondement des industries culturelles repose d'abord sur la nature culturelle des productions, elles sont tout autant marquées par leur nature industrielle et technologique.

\section{DES PRODUCTIONS DE LA REPRODUCTION}

Cette nature industrielle tient tout d'abord au fait que les industries culturelles sont, substantiellement, des industries de la reproduction. Elles se sont d'emblée définies par la capacité de reproduire, via la technologie, des œuvres et des créations singulières: reproduction physique par la fabrication de supports identiques (livre, disque, DVD), reproduction par la multiplicité des sources de projection (cinéma), reproduction par la

\footnotetext{
${ }^{6}$ Il faut d'ailleurs souligner que la situation n'a pas toujours été la même. Dans le modèle « hollywoodien » des studios intégrés, les majors ont ainsi longtemps possédé sous contrat leur propres artistes et techniciens.
} 
transmission généralisée (radio et télévision), reproduction par l'accès permanent (Internet). C'est dans cette capacité de toucher un public nombreux, quasiment sans limite, que réside le cœur de la dynamique économique de ces industries et des conséquences sociales associées, en terme de massification de la consommation notamment.

La capacité de reproduire les biens culturels les plongent en effet dans une économie de l'immatériel aux rendements croissants. Pour résumer, l'essentiel des investissements nécessités par ces biens portent sur la production des œuvres; il est d'autant plus intéressant de stimuler une diffusion massive que les coûts de distribution ne seront pas multipliés dans les mêmes proportions : une fois un film tourné, il ne coûte (presque) rien de plus au producteur (quand c'est possible) de multiplier les salles ou de laisser le film plusieurs semaines de plus à l'affiche. Les industries culturelles s'inscrivent de ce fait dans une économie particulière où les économies d'échelle ne sont quasiment pas envisageables au stade de la production (une industrie de prototypes), mais elles sont par contre rendues presque sans limite par les possibilités de reproduction et peuvent facilement pousser à une saturation du marché (économie de l'anti-rareté).

Ces caractéristiques économiques engagent toutefois la culture dans une spirale infernale susceptible de remettre en cause ces caractéristiques initiales : les économies d'échelle et la massification de la distribution incitent au développement d'actions promotionnelles et commerciales qui favorisent et sont seulement à la portée des producteurs disposant du plus de moyens et les produits visant les marchés les plus larges. Elle renforce de ce fait la banalisation et la standardisation des produits, même quand ils sont ancrés dans un terreau culturel spécifique? ${ }^{7}$.

\section{DES I NDUSTRI ES A BASE DE TECHNOLOGI ES}

Le poids de la reproduction comme caractéristique centrale des industries culturelles explique pour une large part le rôle important du facteur technologique dans son économie. A I'heure où les développements récents de l'Internet sont envisagés avec beaucoup de crainte de la part de la plupart des professionnels de la culture, il est en particulier intéressant de rappeler que tout au long de leur histoire, les industries de la culture se sont régulièrement saisis ${ }^{8}$ des nouvelles technologies pour redynamiser leur filière et s'ouvrir de nouveaux marchés. Que ce soit dans le cinéma, le livre ou le disque, l'arrivée de nouveaux supports de diffusion ou de production a ainsi régulièrement permis une revalorisation profonde des fonds de catalogue. L'industrie phonographique offre l'exemple le plus manifeste d'une telle capacité à surfer sur les innovations techniques pour soutenir son marché. Le passage aux 78t, puis aux microsillons, puis aux cassettes enregistrées, et plus récemment aux $C D$ a permis de relancer périodiquement les ventes en incitant les consommateurs à renouveler leurs discothèques; parallèlement, même si elle a craint, pendant un temps, la concurrence de radios transmettant « gratuitement », l'industrie phonographique a également su se saisir de cette opportunité de promotion et caisse de résonance pour la musique enregistrée. L'exemple de la musique n'est pas isolé. Le livre a également su trouver, avec le développement du brochage, puis des poches, l'occasion de développer très fortement un nouveau marché pour ses ouvrages de catalogue. De même, en dépit, là encore, des craintes longtemps exprimées à l'égard de la télévision, le cinéma a finalement trouvé, avec le développement des chaînes, un second marché extraordinaire pour la valorisation de ses films.

\footnotetext{
${ }^{7}$ C'est par exemple le cas d'Harry Potter.

${ }^{8}$ Même si c'est souvent avec retard et réticence
} 
Ce poids de la fonction technologique de reproduction dans l'équilibre économique de ces industries donne une importante d'autant plus grande aux firmes qui contrôlent les technologies et donc les modes de circulation des produits, en étant à même d'y consacrer les investissements nécessaires : qu'il s'agissent des supports physiques (CD), des terminaux à disposition des consommateurs (lecteurs de supports ou de consultation), des infrastructures de réseaux. Cela explique que dans presque tous les secteurs des industries culturelles, les évolutions marquantes ces dernières années ont été le fait d'une intrusion massive des porteurs de ces technologies: c'est notamment le cas dans la musique, l'audiovisuel et aujourd'hui l'internet. Désormais, les opérateurs de télécommunications et de réseau, les éditeurs et constructeurs informatiques (Microsoft) et de matériels (Sony) disposent de moyens considérablement plus importants que les « majors » de la culture et sont à même de peser très directement sur l'orientation et le développement des filières culturelles ${ }^{9}$.

\section{DES STRATEGI ES I NDUSTRI ELLES CLASSI QUES}

Malgré l'absence d'économies spontanées d'échelles en production, il est intéressant de noter que les industries de la culture ont malgré tout su développer des stratégies industrielles très classiques en travaillant leurs création pour échapper à leur singularité et à leur unicité.

L'enjeu majeur consistait en particulier à échapper aux risques inhérents à chaque création en fidélisant les consommateurs, si ce n'est en les contrôlant, et en inscrivant chaque production dans un cadre plus large et plus stable. Le travail important opéré autour de la construction de marques (en télévision comme dans l'édition) et de collections (notamment dans l'édition) a notamment permis aux entreprises du secteurs d'échapper à l'unicité intrinsèque des œuvres en exploitant des filons et en opérant un passage à la série, jouant à la fois de la standardisation (dans le cadre d'un collection) et de l'individualisation (de chaque composant de la collection).

Du point de vue strictement économique, l'intérêt de telles stratégies tient à la capacité d'opérer des compensations entre produits en globalisant les risques des différents projets (raisonnement en gamme, dans une logique de collection), en maîtrisant les dérapages éventuels sur chaque opération, en visant l'équilibre dans la durée (plutôt qu'au coup par coup), en répartissant les frais de structure. L'objectif est double : augmenter les ressources en valeur absolue et les étaler dans le temps.

Ces stratégies permettent donc d'équilibrer recettes de courts et de longs termes, en déployant, le cas échéant, des actions diversifiées sur les deux registres. Ainsi, il est intéressant de noter que, dans le champ de l'édition, les entreprises développent d'un côté la valorisation sur le long terme, de leur patrimonial éditorial. Pour ce faire, elles stimulent à la fois la cession de droits (adaptations audiovisuelles par exemple) et la multiplication de rééditions ou de compilations ${ }^{10}$. D'un autre côté, il faut cependant relever que ces mêmes éditeurs mettent parallèlement l'accent sur l'optimisation de leurs recettes de court terme en développant des investissements et en rationalisant leur gestion dans la production, la logistique ou la stratégie commerciale : l'accélération des flux d'ouvrages, l'optimisation de leur rotation, l'augmentation du prix pour maintenir le chiffre d'affaires dans un contexte

\footnotetext{
${ }^{9}$ Ce poids est d'autant plus important que les industries de la culture n'ont, traditionnellement, que peu investi dans la recherche et développement et la maîtrise technique de leurs supports.

${ }^{10}$ Elles représentent, dans le cas du livre, la moitié de la production globale en nombre de titres
} 
baissier leur permet ainsi de produire davantage, à moins d'exemplaires, proposés moins longtemps et payés plus cher.

\section{DES INDUSTRI ES DE DISTRIBUTI ON}

Les rapports économie et culture se présentent et se posent spontanément au niveau de la production car les modes industriels de production ont une incidence directe sur la nature, la qualité et la richesse des œuvres produites. Par contre, ce sont les structures de diffusion et distribution qui pèsent le plus sur les industries culturelles. Le constat s'opère très facilement en examinant le rapport des structures de coût entre production et diffusion. Quelque soit le secteur concerné, de la moitié aux deux tiers du prix payé par le consommateur pour un bien culturel est consacré à la rémunération des intermédiaires de diffusion.

Plusieurs facteurs expliquent ce poids de la distribution dans les industries culturelles ${ }^{11}$. La première raison tient ben sûr aux rendements croissants très importants ouverts par la reproduction des œuvres. II s'explique aussi par la nécessité, même dans le cadre d'une économie de l'immatériel, d'assurer la distribution physique des œuvres (transport et mise à disposition des supports, gestion des infrastructures de diffusion). Une troisième raison tient aux caractéristiques informationnelles du marché des biens culturels: grande variété et information incomplète du consommateur. Comme nous avions eu l'occasion de le souligner à propos de l'édition littéraire ${ }^{12}$, la diffusion et l'accès aux produits culturels relève en effet souvent d'une équation impossible: pour le livre, ce sont 200000 titres disponibles, édités par 4000 éditeurs différents, à distribuer dans 26000 librairies et points de vente potentiels !

La résolution de cette équation s'est progressivement traduite par une montée en puissance des intermédiaires de programmation et de distribution, observable, sous une forme ou une autre dans tous les secteurs. Le premier effet, que les économistes expliquent volontiers par la théorie du monopole naturel, est la concentration des réseaux : l'importance des coûts d'infrastructure rend économiquement peu rentable la constitution de réseaux de distributions concurrents et favorise au contraire leur partage ou leur mutualisation. Le phénomène engagé depuis plusieurs années dans le cinéma et le disque, s'est accéléré récemment aussi bien dans le livre, que dans la télévision ${ }^{13}$. Ce développement des distributeurs s'est accompagné également du renforcement du poids des grandes surfaces, spécialisées ou non, pour lesquels les biens culturels représentent un facteur d'attractivité des consommateurs et un produit à haute valeur ajoutée.

La seconde façon de résoudre cette équation est passée par une réduction drastique de la diversité et la distribution qui a eu une influence importante en terme d'homogénéisation culturelle. Cette concentration sur un nombre toujours réduit d'œuvre s'observe dans la musique mais aussi dans le cinéma, la littérature ou la télévision : dans chacun de ces cas, alors que le volume des œuvres produit ou diffusé tend à augmenter, le nombre d'œuvre contribuant à la fréquentations, l'écoutes ou au chiffre d'affaires tend parallèlement à diminuer. Alors que les exigences de la production nécessitent, pour assurer le renouvellement des œuvres et trouver de nouveaux succès, une diversité des artistes, des cultures et des ressources créatives, les contraintes de la diffusion de masse fait que cette diversité est, paradoxalement, réinterprétée dans un modèle de consommation appelant banalisation et homogénéisation. Les partenariats toujours plus serrés entre éditeurs phonographiques et média audiovisuels aboutissent par exemple à une réduction drastique

\footnotetext{
${ }^{11}$ Benghozi, P.-J . et T. Paris (2005). Analysing the Distribution to Understanding the Markets of Cultural Goods. 8 th International Conference on Arts \& Cultural Management. HEC-Montréal

${ }^{12}$ Benghozi, P.-J . et C. Moire (1987). "Petit éditeur peut-il devenir grand?" Gérer et Comprendre 8: 18-27.

${ }^{13} \mathrm{Cf}$. La fusion à l'ordre du jour entre TPS et Canal Satellite
} 
de la diversité des œuvres au profit des succès les plus porteurs et permet aux grande surfaces de ne proposer qu'un nombre réduit de références ${ }^{14}$.

\section{Dynamiques économiques et stratégiques}

Pour comprendre les mutations et articulations contemporaines des industries culturelles, il est, paradoxalement, essentiel tout d'abord de garder en permanence à l'esprit que ces industries sont anciennes, parfois pluriséculaires, et que leur économie s'inscrit dans la durée. C'est ainsi que les structures existantes aujourd'hui, les principes à l'œuvre dans les modèles d'affaires, les règles régissant le droit d'auteur, et même certaines stratégies commerciales telles que diffusion de masse et best sellers se sont mis en place très tôt dans I'histoire de ces industries. Ils contribuent à éclairer très directement les situations observables aujourd'hui ainsi que les stratégies et réponses spontanément mises en oeuvres (Rouet 1989; Nicolas 1996; Paris 2002).

Une des premiers manifestations de cette ancienneté des industries culturelles est sans doute leur immuabilité apparente et leur difficulté à reconstruire de nouveaux modèles économiques. La seconde manifestation est un effet de vieillissement qui frappe toutes ces industries et les conduit justement à redéfinir régulièrement leurs principes de fonctionnement (Diberder 1985). Même un secteur récent comme celui de la télévision a ainsi été frappé par une saturation de son marché une fois l'ensemble du territoire couvert d'antennes, les foyers équipés de deux ou trois postes, les programmes diffusés quasiment en continu, les espaces publicitaires à leur maximum à certaines heures et les téléspectateurs passant un temps toujours plus long devant leur poste ${ }^{15}$. Mais cette saturation n'a pas empêché, dans le même temps, un profond renouvellement de la filière avec l'ouverture au privé, le développement de nouvelles techniques de transmission et de production permettant une démultiplication des chaînes et des programmes, I'internationalisation du marché ouvrant à de nouveaux programmes et en réduisant les coûts, et surtout une évolution historique des modèles d'affaires faisant une large place au paiement des opérateurs par le spectateur (sous forme d'abonnements notamment, mais aussi de SMS, de produits dérivés...), le transformant de téléspectateur en consommateur ${ }^{16}$. De façon analogue, la situation particulièrement dynamique d'un « vieux » média tel que la radio attestent des fortes capacités de redynamisation des industries de la culture, en se réorientant, en l'occurrence, à partir de la diffusion musicale plus que du développement d'un modèle éditorial de type presse.

Ce sont quelques une de ces lignes de force et de renouvellement que nous allons maintenant évoquer dans la suite de cette communication.

\section{UNE SURPRODUCTI ON GENERALI SEE}

A l'image de la télévision étendant progressivement la durée de ses programmes et le nombre de ses chaînes, un des constats qui émergent spontanément, lorsque l'on examine l'ensemble des secteurs des industries culturelles, est celui d'une surproduction généralisée. Le disque comme le livre montrent l'un comme l'autre un nombre d'albums en augmentation et une évolution spectaculaire du nombre de jeunes talents. Si le cinéma est resté, de son

\footnotetext{
${ }^{14}$ aussi bien pour le disque que pour le livre. seules moins de 5000 références sont proposées en hypermarchés contre de l'ordre de 200000 références possibles.

${ }^{15} 3 \mathrm{~h} 30$ contre à peine $2 \mathrm{~h}$ en 1980

${ }^{16}$ Diberder, A. L. et N. Coste-Cerdan (1986). La télévision. Paris, La Découverte
} 
côté relativement stable depuis de nombreuses années quant au nombre de films produits, c'est que le chiffre était déjà haut (alors même que la fréquentation a longtemps dramatiquement chuté) avec un nombre de premiers films à un niveau lui même exceptionnel (plus de 40\%). Si l'on peut parler de surproduction à ces égards, c'est que, sauf dans certains cas, la croissance importante de la production ne correspond pas, loin de là, à une évolution correspondante de la consommation culturelle. L'exemple du livre montre plutôt que l'évolution importante de la production s'est accompagnée parallèlement d'une réduction importante des tirages.

Le haut niveau de la production semble plutôt correspondre au développement de ce que nous appelions plus haut une économie-casino. Les producteurs spéculent en lançant de plus en plus d'œuvres, dans l'espoir que l'une d'elles leur permettra de décrocher le gros succès, quitte à abandonner d'autant plus rapidement celles qui ne trouve pas rapidement leur public: à l'image des centaines de romans lancés à chaque rentrée littéraire et souvent oubliés à peine quelques semaines après.

On constate ainsi un double mouvement - concentration croissante des recettes / multiplication de l'offre - rejoignant ce que les économistes ont qualifié de star system (Rosen 1981; Benhamou 2002). La massification de la diffusion provoque le découplage entre le succès de quelques produits-stars de moins en moins nombreux et la multiplication des échecs pour les autres. Le phénomène est très général et se voit encore renforcé avec le développement de l'internet. II est d'autant plus fort qu'il se situe au point de convergence des stratégies de producteurs, des distributeurs et des médias. Comme nous l'avons montré par ailleurs (Benghozi et Paris 2003), dans ce contexte de concentration/ multiplicité, les prescripteurs (distributeurs et programmateurs notamment) prennent un rôle et un poids croissant en orientant les choix des consommateurs, par le choix et la mise en avant qu'ils opèrent sur certaines oeuvres.

La concentration des productions, des droits de propriété, des médias et circuits de diffusion et des structures de distribution s'opère dès lors à tous les stades des industries culturelles pour capturer l'audience, contrôler le marché et stabiliser les recettes, élever les barrières à l'entrée pour les concurrents potentiels. Cette situation pose directement, au niveau national tout autant qu'international, la question du plurarisme des formes d'expression artistiques et culturelles accessibles (et non pas seulement produites). Le risque principal d'uniformisation des contenus ne tient pas aujourd'hui à une réduction de la production mais essentiellement à une focalisation sur les œuvres les plus attractives et à un marché à deux vitesses entre produits marketés ou à diffusion restreinte.

Au delà de cette focalisation sur un petit nombre de créations, la logique du star system a, du point de vue économique, deux effets induits importants.

Le premier relève d'une spirale inflationniste des coûts. Au fur et à mesure que l'écart se creuse entre quelques œuvre à (très gros) succès et une grande majorité d'échecs ou de recettes très moyennes, l'ensemble des acteurs la filière est incité à développer les coûts associés à chaque oeuvre pour espérer pouvoir mieux se distinguer et accéder au petit nombre des produits star. Les surcoûts affectent autant la production que la distribution, la promotion et la publicité ${ }^{17}$.

\footnotetext{
${ }^{17}$ Les coût de publicité tendant même à augmenter de façon proportionnellement considérablement plus forte que ceux de production
} 
Le second effet concerne la segmentation qui s'opère entre les œuvres et leurs modèles économiques. Le star system pousse en effet à la valorisation maximale des recettes et des droits dérivés, dans une logique du winner take all. A l'image de ce que l'on peut observer pour les droits sportifs télévisuels, il s'établit dès lors une coupure entre des œuvres à succès pour lesquels diffuseurs et consommateurs sont prêts à payer, même très cher, et des œuvres qui ne trouvent pas leur public et acceptent parfois des conditions de diffusion sous forme de quasi gratuité, dans l'espoir d'avoir au moins une visibilité et un accès au public: c'est le cas pour les ouvrages bradés chez les soldeurs comme pour les musiciens mettant leur morceaux en libre téléchargement.

Les différentes dynamiques que nous avons pointées (surproduction, montée en puissance des diffuseurs, concentration de la consommation sur un nombre d'œuvres de plus en plus restreint, émergence de nouveaux acteurs et évolution des formes de concurrence) se sont accompagnées d'une accentuation de la fonction de programmation, assumée notamment par des distributeurs agrégateurs de la demande et prescripteurs dans les choix des consommateurs. En choisissant de mettre en avant un nombre restreint de produits, ou au contraire une offre la plus large possible, en choisissant de mettre en place des relations ponctuelles avec les consommateurs ou en établissement au contraire des relations suivies par abonnement, les distributeurs sont conduits, sans l'avoir toujours anticipé, à faire évoluer leur métier et leur position dans la filière : en assumant un rôle plus ou moins affirmé de prescripteur, et en s'engageant plus ou moins en amont dans la chaîne de valeur. Comme nous l'avons montré par ailleurs (Benghozi et Paris 2005a; Benghozi et Paris 2005b), ce sont bien autour de ces fonctions de prescriptions que se réorganisent aujourd'hui les modèles d'affaires des industries culturelles.

\section{DES MARCHES DE PLUS EN PLUS DIVERSI FI ES ET SEGMENTES}

Au delà des constats généraux qui peuvent être dégagés des dynamiques à l'œuvre dans la culture, il est tout aussi important de rappeler qu'aucune règle générale ne peut cependant être tirée quant aux arrangements spécifiques auxquels donnent lieu les industries de la culture en France et dans le monde. La grande variabilité des situations et des solutions organisationnelles est essentielle à garder à l'esprit alors même que l'ensemble des professionnels a au contraire généralement tendance à globaliser les situations en ignorant les spécificités des différents marchés et sous-marchés de la culture. Nous avions déjà montré que même le droit d'auteur, pourtant généralement défendu sur la base de principes génériques forts faisait, en pratique, l'objet d'applications très différentes dans la musique, le cinéma, le livre ou les arts plastiques par exemple (Benghozi et Paris 1999). Ce cas n'est pas isolé et la confrontation des différentes industries conduit à relativiser nombre d'assertions souvent présentées comme allant de soi : le poids de la mondialisation et la domination américaine dans les industries les plus internationalisées par exemple. Ainsi, si le cinéma constitue effectivement un marché très largement contrôlé par les USA, il est paradoxal de constater que la situation de la musique est bien moins tranchée. On observe en effet dans ce cas, un marché certes en oligopole, mais plutôt bien équilibré entre Europe, USA et Japon; en outre, si la domination économique d'une variété « internationale » banalisée est avérée en production, il existe malgré tout une bonne résistance des marchés locaux $(60 \%$ des ventes en France relève de musique d'expression française), sans commune mesure avec celle existant dans le cinéma alors que la musique se prête spontanément plus facilement aux échanges (pas de nécessité de doublage).

On observe cette même variabilité des situations dans chacun des grands secteurs des industries de la culture. A partir de principes économiques partagés, chacun d'eux s'organise en autant de sous-marchés. Cette configuration tient au déploiement de stratégies 
différenciées et au partage de certaines caractéristiques communes des produits créés et échangés.

La structuration de ces sous-marchés tient d'une part à l'existence de canaux diversifiés de diffusion des œuvres et au positionnement différencié des acteurs économiques sur ces canaux. Le cas du cinéma nous montre par exemple que la possibilité de valoriser les films à la fois dans les réseaux de salles, sur les canaux de télévision et dans la distribution éditoriale vidéo et DVD conduit à une segmentation des productions en trois grands marchés (blockbusters, films «amphibies» de télévision, films d'auteurs et films de genre) correspondant à la coexistence de modèles complémentaires sensiblement différents de financement, de valorisation et de conception ${ }^{18}$. II s'établit ainsi un équilibre entre les différents supports de l'audiovisuel : salles, câble, satellite, chaînes hertziennes, éditions DVD... Loin d'y voir des marchés en concurrence, il faut de plus en plus y voir des marchés en complémentarité veillant à positionner chacun des supports de façon spécifique en évitant leur cannibalisation réciproque ${ }^{19}$ et dont les développements s'appuient l'un l'autre.

Un deuxième facteur de structuration des sous-marchés tient au partage, pour une gamme de produits, de certaines caractéristiques spécifiques. C'est ainsi que pour comprendre l'économie de l'édition littéraire aujourd'hui, il faut dépasser le modèle général de la littérature générale qui vient spontanément à l'esprit quand on évoque ce secteur. En effet, l'édition de livres s'organise aujourd'hui autour de plusieurs marchés très différents dans leur économie et leur structure: littérature générale certes, mais aussi BD, jeunesse, livres scolaires, beaux-arts, ouvrages pratiques et ouvrages scientifiques notamment. Chacun d'eux correspond à des modèles d'affaires très différents quant à la structure et aux coûts de production, à la nature et au degré du risque éditorial, à la fréquence de production et aux tirages, à l'internationalisation... Le cas de musique montre enfin encore plus nettement comment des stratégies parfois globales peuvent se déployer aussi autour de la structuration de genres musicaux correspondant à des communautés élargies de labels, de médias, d'artistes et de public.

De telles formes de structuration constituent, à bien des égards, un principe d'organisation particulièrement fort dans le domaine culturel, ainsi que le montre les développements récents dans I'Internet (Gensollen 1999). La multiplicité des expressions culturelles a d'ailleurs sans doute été à la base de son développement récent : la forte croissance des sites et des réseaux a en effet été portée, au début, par la multiplication de communautés virtuelles spontanées traduisant des formes d'agrégation et structuration sociale labile et évolutive, s'appuyant sur centres d'intérêts et cultures communs. L'organisation des industries créatives a ainsi été envisagée par certains auteurs comme le développement d'interactions globales dans un réseau et des communautés, notamment dans le cadre d'une proximité géographique (Brown et Duguid 2000; Landry 2000; Santagata 2002).

Les modèles de développement des industries culturelles sont donc d'autant plus intéressants à relever qu'ils sont particulièrement en phase avec une économie de l'internet qui s'appuie justement sur l'opérationnalisation et la marchandisation des communautés d'échanges et de pratiques (Rheingold 1993). Le développement des échanges P2P en est une des formes d'expression particulièrement symptomatique, mais c'est loin d'être la seule.

\footnotetext{
${ }^{18}$ Benghozi, P.-J . (1989). "Films évènements et téléfilms: les deux faces de l'économie du cinéma." Problèmes Economiques 2143 - 4(10-89)

19 cf; les débats sur la "troisième fenêtre" visant à prévoir la commercialisation sur chaîne satellite en organisant de façon concertée et organisée la temporalité de la commercialisation des films de cinéma sur différents supports : salles, vidéo après 9 mois, chaîne payante après un an, chaîne généraliste après deux ans...
} 


\section{DES STRUCTURES DE CONCURRENCE COMPLEXES ET EVOLUTI VES}

Pour la plupart des biens marchands, le prix reflète pour une part les coûts de fabrication et la qualité et permet donc, de fait, une segmentation des marchés. Dans le cas des biens culturels par contre, comme nous l'avons déjà noté, le prix de référence est un prix de distribution indépendant des structures de production. Dès lors une concurrence frontale est à même de s'opérer entre production correspondant à des modes d'organisation et de création radicalement différent. Sur les écrans d'un multiplexe, le blockbuster sera projeté à côté d'un petit film d'auteur, tout comme, sur les rayons d'une librairie, un premier roman longuement mûri et publié chez un petit éditeur côtoieront les mémoires de commande d'une star people publiées chez un éditeur de renom. Une conséquence de la double dimension créative et industrielle des industries de la culture réside donc dans la superposition et l'articulation permanente de modèles économiques différents: dans les mêmes filières industrielles et sur les mêmes marchés.

Cette situation recouvre, plus généralement, une forme de concurrence entre majors et indépendante que l'on retrouve dans l'ensemble des industries culturelles. Elle s'avère d'autant plus complexe à analyser qu'elle s'accompagne également de formes de partenariats parfois très forts. Les majors ont besoin des indépendants pour leur capacité de repérage des nouvelles tendances créatives, et faute de disposer des moyens financiers et des infrastructures, les indépendants font régulièrement appel aux majors pour coproduire ou distribuer leurs produits. Cette situation est à la base de solidarités parfois inattendues. Elle pose également des difficultés considérables du point de vue de la régulation et de l'action publique. L'histoire récente de l'édition littéraire, lors de l'éclatement d'Editis et du projet de rachat par Hachette, illustre l'un et l'autre de ces deux points. Elle témoigne en outre de la contradiction qu'il peut exister entre le souci de maintenir une diversité des structures de production dans le cadre national et celui de défendre, au niveau mondial, une spécificité culturelle française portée par la constitution d'un acteur national puissant dans une économie mondialisée.

Un autre facteur de complexité des structures de concurrence à l'œuvre dans les industries de la culture tient à la montée en puissance de nouveaux acteurs, notamment des offreurs de technologie. Les industries culturelles sont fortement attractives pour les consommateurs et mobilisées par des industries plus traditionnelles pour "enrichir » des offres plus traditionnelles. Cette instrumentalisation de la culture se traduit d'une part par une intensification des partenariats entre «producteurs » et diffuseurs, les œuvres et les artistes étant utilisés comme des produits d'appel pour appuyer des formes renforcées de commercialisation ${ }^{20}$. Mais les produits culturels sont aussi, d'autre part, mobilisés de façon plus profonde et structurelle dans une économie désormais de plus en plus immatérielle, à base de connaissance et de contenus. C'est le cas en particulier dans le développement de I'Internet et des industries des télécommunications qui nécessitent - pour élaborer des sites comme pour attirer les consommateurs - images, sons, musique, graphismes, textes...

Cette présence grandissante de nouveaux acteurs, extérieurs aux filières traditionnelles de la culture sape les bases traditionnelles du secteur en bouleversant l'organisation et l'équilibre

\footnotetext{
${ }^{20}$ On fait couramment remonter cette stratégie à l'apparition de la vidéo domestique et à l'échec rencontré par Sony dans la commercialisation de son standard (Betamax) au profit de celui de JVC (VHS). En dépit d'une moindre qualité et d'une réputation a priori moins favorable, les partenariats noués avec les majors hollywoodiennes permirent à JVC de proposer les grands films américains exclusivement en VHS et représentèrent un facteur d'attraction radical pour les consommateurs. Les investissements ultérieurs de Sony dans les studios de cinéma et l'édition musicale peuvent s'interpréter comme une réponse visant à garantir la disponibilité des contenus pour les nouveaux supports de consommations développés.
} 
économique des filières (capacités d'investissement), en creusant l'écart entre structures de production et structures de distribution, et en dissolvant les traditionnelles frontières des secteurs de la culture. Aujourd'hui, les opérateurs de télécommunications et de réseau, les éditeurs et constructeurs informatiques (Microsoft) et de matériels (Sony) disposent de moyens considérablement plus importants que les «majors » de la culture et sont à même de peser très directement sur l'orientation et le développement des filières culturelles. Les contraintes économiques auxquelles obéit désormais la culture ne sont donc plus simplement celle de l'«économie de la culture», mais aussi celle d'une économie plus vaste dans laquelle la culture ne constitue qu'un élément parmi d'autres et n'est donc pas à même de peser sur ses grandes orientations.

Sous l'effet de ces nouveaux acteurs, les industries culturelles ont ainsi vues leur cadre s'élargir, ces dernières années, notamment vers des secteurs qui n'étaient pas toujours conçus précédemment relevant du domaine créatif : jeu vidéo, design, téléphonie mobile par exemple. Cet effet s'est également vu renforcé par une dynamique interne de diversification et d'élargissement de leurs ressources (Benghozi 1995). La possibilité de revenus annexes importants a modifié sensiblement les phases de conception et de réalisation (pour intégrer, dès que possible, la possibilité de produits dérivés) et celle de distribution (pour renforcer la promotion et les effets de notoriété). En faisant intervenir très tôt, dans le financement comme dans la définition d'une oeuvre, l'ensemble des partenaires qui contribueront à son développement et à sa diffusion, la marchandisation remet en particulier en cause l'organisation traditionnelle de la production. Ainsi, les producteurs de disques ou les chaînes de télévision ne préparent plus seulement une chanson, un album ou un programme de variétés, ils conçoivent d'emblée un tout cohérent comprenant singles successifs, album, clips, affiches, tournée de concerts, magazines spécialisés, émissions radio ou télé, produits dérivés ( $T$ shirts, collectors), sonneries et logos téléphoniques, sites web...

\section{Conclusion : des principes d'action à trouver pour les politiques publiques}

Les spécificités industrielles et culturelles rendent particulièrement sensibles et difficiles la régulation et la gouvernance des échanges culturels au niveau national aussi bien que mondial. En effet, les effets éventuels de domination économique ne soulèvent pas simplement, comme dans d'autres secteurs, des questions de tarification, de prix d'accès, voire même de liberté du consommateur ou de diversité de l'offre. Ils affectent aussi très directement la vie, l'identité et l'intégrité même des individus, dans ce qu'ils ont de plus intime.

Les politiques publiques en matière d'industries de la culture sont le plus souvent décalquées de modalités traditionnelles d'intervention dans la culture, mais essentiellement marquées par le souci de promouvoir la création. Ces interventions rencontrent, à ce titre, des difficultés qui ont été notées depuis longtemps (Rouet 1991) : niveau et modalité des actions engagées, stade de la filière visé et nature des bénéficiaires, choix et définition des instruments d'intervention, conditions de l'évaluation et de l'éligibilité. L'action publique se heurte toutefois à des difficultés d'autant plus grandes que les enjeux économiques et marchands de ces industries apparaissent plus importants. Deux dimensions nous semblent mériter, de ce point de vue, d'être soulignées. La première concerne la capacité de définir des interventions ciblées dans des secteurs dont les frontières se recomposent sous l'effet de la diversification des produits culturels et de la convergence des technologies. La seconde, 
plus fondamentale, relève de la légitimité même d'une intervention publique ayant pour ambition de corriger des déséquilibres économiques dans la sphère marchande au nom de politiques culturelles dont les objectifs sont, par nature, difficile à définir et à mettre en cohérence.

Ces difficultés trouvent toute leur ampleur au niveau international. La régulation mondiale a essentiellement été pensée, jusqu'ici dans le cadre d'instances internationales reposant de fait sur un principe de régulation interétatique conçu comme prolongement des politiques nationales. Or, l'aboutissement des logiques industrielles de la culture fait émerger et consolide des marchés extranationationaux contournant les réalités et régulations nationales. Dans cette configuration, faute de structures de régulation publiques adaptées, le seul niveau d'intégration et d'action supranational relève de fait d'opérateurs privés multimédias face auxquels les outils nationaux d'intervention se révèlent inadaptés.

Certes, la mondialisation des échanges a toujours existé en matière culturelle, mais elle reposait jusque là sur des échanges commerciaux réellement équilibrés ou dans une dissymétrie assumée. Une des nouveautés actuelles est sans doute que la mondialisation repose aujourd'hui sur un postulat d'égalité, qui peut être facteur d'espoir mais masque en pratique une inégalité de fait liée à l'existence de ressources et de poids différents de la part des multiples acteurs des filières culturelles. 


\section{Références}

Benghozi, P.-J. (1989). "Films évènements et téléfilms: les deux faces de l'économie du cinéma." Problèmes Economiques 2143 - 4(10-89).

Benghozi, P.-J. (1995). "La diversification des productions culturelles." Revue Française de Gestion 106(Novembre-Décembre): 65-75.

Benghozi, P.-J. et C. Moire (1987). "Petit éditeur peut-il devenir grand?" Gérer et Comprendre 8: 18-27.

Benghozi, P.-J. et T. Paris (1999). "Authors' Rights and Distribution Channels: an Attempt to Model Remuneration Structures." International Journal of Arts Management 1 Spring(3): pp.44-58.

Benghozi, P.-J. et T. Paris (2003). "De l'intermédiation à la prescription: le cas de la télévision." Revue Française de Gestion 142(Janvier-Février): 205-227.

Benghozi, P.-J. et T. Paris (2005a). Analysing the Distribution to Understanding the Markets of Cultural Goods. 8th International Conference on Arts \& Cultural Management. HECMontréal, François Colbert.

Benghozi, P.-J. et T. Paris (2005b). The Economics and Business Models of Prescription in the Internet. Internet Economics. E. Brousseau et N. Curien, Cambridge University Press.

Benhamou, F. (2002). L'économie du star system. Paris, Odile Jacob.

Braczyk, H.-J., G. Fuchs, et al. (1999). Multimedia and Regional Economic Restructuring. London, Routledge.

Brown, J. S. et P. Duguid (2000). The Social Life of Information. Boston, Mass, Harvard Business School Press.

Caves, R. E. (2000). Creative Industries: Contracts Between Art and Commerce. Cambridge, Mass, Harvard University Press.

Chandler, A. D., P. Hagstrom, et al. (1998). The Dynamic Firm: The Role of Technology Strategy Organization and Regions. Oxford, Oxford University Press.

Diberder, A. L. (1985). "Formation du profit dans les industries culturelles." Réseaux(14): pp.34-44.

Diberder, A. L. et N. Coste-Cerdan (1986). La télévision. Paris, La Découverte.

Flichy, P. (1991). Les Industries de l'Imaginaire, Presses Universitaires de Grenoble.

Florida, R. (2002). The Rise of the Creative Class. New York, Basic Books.

Gensollen, M. (1999). "La création de valeur sur internet." Réseaux 97: 15-76.

Greffe, X. et X. Dupuis (1981). "Quand I'opéra découvre la gestion." Revue Française de Gestion 30.

Howkins, J. (2001). The Creative Economy: How People Make Money from Ideas. London, Allen Lane.

Keat, R. (2000). Cultural Goods and the Limits of the Market. New York, St. Martin's Press. Landry, C. (2000). The Creative City: A Tool kit for Urban Innovators. London, Earthscan.

Nicolas, B. (1996). Structures d'organisation et paradigmes sectoriels: le cas des studios de cinéma en France et au Royaume Uni (1895-1995). Gestion. Paris, Ecole polytechnique: 464.

Paris, T. (2002). Le droit d'auteur: l'idéologie et le système. Paris, PUF. 
Rheingold, H. (1993). The Virtual Community: Homestanding on the Electronic Frontier. New York, Addislon-Wesley.

Rosen, S. (1981). "The Economics of Superstars." American Economic Review 71: 845-58.

Rouet, F., Ed. (1989). Economie et culture: Les industries culturelles. Paris, La Documentation française.

Rouet, F. (1991). Le soutien aux industries culturelles en Europe. Paris, La documentation française.

Santagata, W. (2002). "Cultural Districts: Property rights and sustainable economic growth." International Journal of Urban and Regional Research 26: 9-23.

Schussman, A. et K. Healy (2002). Culture, Creativity and the Economy: An Annotated Bibliography of Selected Sources. Tucson, Department of Sociology \& Social Sciences, University of Arizona: 29.

Throsby, D. (2001). Economics and Culture. New York, Cambridge, Melbourne, Cambridge University Press.

Tulgan, B. (2001). Winning the Talent War. New York, Norton. 